\title{
Application of 3-dimensional printing implants for bone tumors
}

\author{
Jong Woong Park, MD ${ }^{1,2}$, Hyun Guy Kang, MD, PhD ${ }^{1,2}$ \\ ${ }^{1}$ Orthopaedic Oncology Clinic, National Cancer Center, Goyang, Korea; ${ }^{2}$ Division of Convergence Technology, National Cancer Center, Goyang, Korea
}

Three-dimensional (3D) additive manufacturing has recently been used in various medical fields. Among them, orthopedic oncology is one that utilizes it most actively. Bone and tumor modeling for surgical planning, personalized surgical instrument fabrication, and implant fabrication are typical applications. The 3D-printed metal implants using titanium alloy powder have created a revolutionary change in bone reconstruction that can be customized to all body areas; however, bioprinting remains experimental and under active study. This review explores the practical applications of 3D printing in orthopedic oncology and presents a representative case. The 3D-printed implant can replace the conventional tumor prosthesis and auto/allobone graft, thereby personalizing bone reconstruction. Biologic bone reconstruction using biodegradable or bioprinted materials beyond metal may be possible in the future.

Key words: Three-dimensional printing, Additive manufacturing, Implant, Limb salvage surgery, Bone tumor

\section{Key message}

- The application of 3-dimensional (3D) printing in orthopedic oncology is summarized into bone and tumor modeling, patient-specific instruments (PSIs), custom-made implants, and tissue engineering.

- The 3D-printed customized implant is the most central application, while modeling and PSI often play adjunct roles.

Short-term surgical outcomes of custom-made 3D-printed implants are promising.

\section{Introduction}

Additive manufacturing (AM), also called 3-dimensional (3D) printing, was conceptually attempted in 1981 by Hideo Kodama, and Charles Hull filed a patent application in the United States in 1986. ${ }^{1-6)}$ There are many types of 3D printers that use various materials. Metals, polymers, hydrogels, and living cells are intended for 3D printing use in the medical field. Metals and polymers are currently being 3D-printed in the medical field. When living cells are printed as organs/organ-like objects, the process is called bioprinting.

AM has 2 advantages that enable its use in the medical field. First, AM enables the manufacturing of shapes that could not be made using traditional production methods. ${ }^{5,7,8)}$ Specifically, a stacking structure with different materials and a planned internal porosity can be created using AM. A lattice structure with a planned internal porosity has unique mechanical properties and provides a scaffold for tissue integration. Moreover, stacking with a hydrogel layer and a living cell layer is the key mechanism of bioprinting. Second, the cost of products fabricated by AM remains constant, even when mass production is difficult because of diversification. ${ }^{9}{ }^{10)}$ To achieve personalized medicine, many medical products must be personalized for each patient, for which AM is suitable.

In the medical field, orthopedic oncology is one that utilizes 3D-printing most actively. Malignant bone tumors can affect any part of the skeleton to various extents with or without joint or growth plate involvement. Moreover, bone tumors, such as osteosarcoma, have a bimodal incidence and more often affect young patients with small bones than adults. In orthopedic oncology, complete bone-tumor excision with safe margins, which provides an appropriate normal tissue envelope around the tumor and enables the reconstruction of massive bone defects, are two of the main steps of limb salvage surgery for malignant bone tumors. Therefore, limb salvage surgery represents a wide variety of surgeries, and all are highly individualized. In orthopedic oncology, modular endoprosthesis with multiple pieces assembled during surgery is most commonly used to insert implants of various sizes. However, modular implants are not available in all circumstances. For example, custom-made implants are required for the reconstruction of unusual sites such as the pelvis or small bones in children. Another major indication for 3D-printing custom-made implants is in cases of anticipated improved surgical results. When a bone tumor is close to the joint or physis, limb salvage surgery using conventional implants is often performed as arthroplasty and sacrifices the joint. However, with a 3D-printed custom-made implant, the surgery can be reduced to segmental reconstruction, which preserves the joint and physis (Fig. 1). Preservation of the adjacent natural joint

Corresponding author: Hyun Guy Kang, MD, PhD. Orthopaedic Oncology Clinic, Center for Rare Cancers, Research Institute and Hospital, Graduate School of Cancer Science and Policy, National Cancer Center, 323 IIsan-ro, Ilsandong-gu, Goyang 10408, Korea 


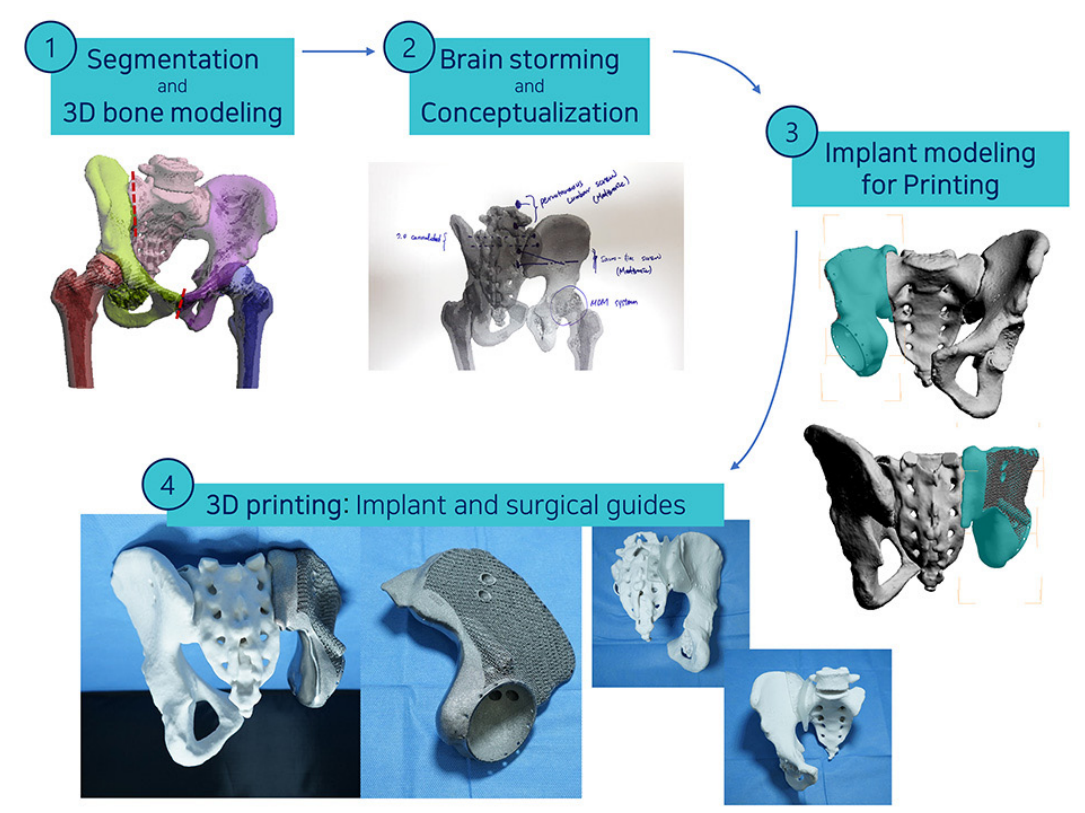

Graphical abstract. Flow chart of the 3-dimensional (3D)-printing of the personalized surgical instrument and implant fabrication in orthopedic oncology.

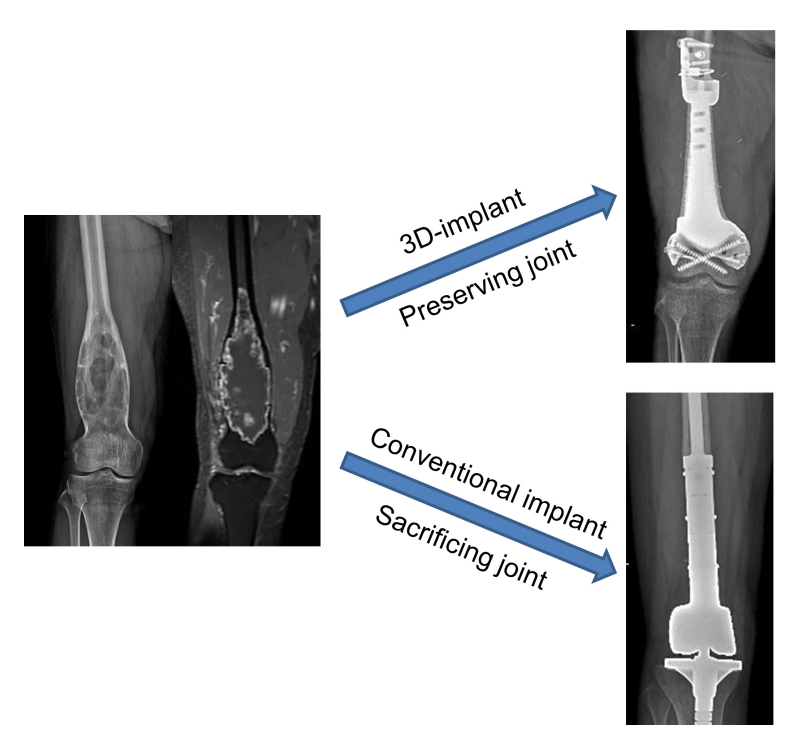

Fig. 1. Representative indication for use of a 3-dimensional (3D)-printed custom-made implant. The plain radiograph and gadolinium-enhanced T1-weighted magnetic resonance image shows chondrosarcoma of the right distal femur. Anticipated surgical results are shown as segmental reconstruction using 3D-printed custom-made implant (right upper) and arthroplasty using a conventional tumor prosthesis (right lower).

is functionally beneficial, ${ }^{11)}$ while preservation of the growth plate reduces gradual postoperative limb length discrepancies.

To summarize the medical application of 3D printing for the treatment of bone tumors, this review focuses on the current clinical applications by usage type. The 3D-printing technology is being applied to bone-tumor surgery in various ways, such as surgical planning through preoperative bone-tumor modeling, fabrication of bone-replacing implants, and personalized surgical instruments. ${ }^{12)}$ To enhance understanding, here we present full treatment flows for young patients with malignant bone tumors who underwent limb salvage surgery.

\section{D printers and materials in orthopedic surgery}

The International Standard Organization defines 3D printing as the "fabrication of objects through the deposition of a material using a print head, nozzle, or another printer technology." In other words, 3D printing is an AM process that involves layerby-layer stacking. The main methods are based on powder solidification, liquid solidification, and extrusion. $\left.{ }^{6}\right)$ Each 3D printer type has its own advantages and disadvantages, and the appropriate materials for printing are limited.

For clinical application in orthopedics, a few types of 3D printers with appropriate properties are commonly used. For bone modeling, various polymers or plaster can be utilized by any type of 3D printer if only a certain strength and precision are obtained. It is technically demanding to implement textures in simulated surgery, distinguish colors for selective anatomical structures, or use transparent materials. However, 3D-printed models, which are made of simple and single materials, are often sufficient in orthopedics. Unlike modeling, for surgical instruments or implants that are in contact with or placed in the surgical field, materials and 3D printers are subjected to strict regulations and certifications. Biocompatibility and sterilizing properties are important for their use as materials for surgical guides, the so-called patient-specific instrument (PSI). Biocompatible resins such as MED610, nylon, and polyamide have been utilized for PSI. ${ }^{13,14)}$ The fabrication of 3D-printed custom-made implants is limited by stricter regulations than those for PSI. Titanium alloy is the most widely used material for producing custommade implants by 3D printing. Metal printing for medical use is usually of the powder-based type, and common printer types 
include electron beam melting and selective laser melting, whose energy delivery vary. ${ }^{15-22)}$ In the field of bioprinting and tissue engineering, 3D printing is still in the experimental stage, and various standard or custom-made $3 \mathrm{D}$ printers are used.

\section{Clinical application in orthopedic oncology}

\section{Bone and tumor modeling}

In many medical fields including orthopedics, visualization using a 3D-printed model is an easy and simple method. Before arthroplasty for severely destructive or deformative joints, bone modeling provides direct visualization of bone defects or deformities and opportunities for simulated surgery, including bone reaming and implant sizing and positioning. 23-27)

In orthopedic oncology, 3D-printed models are also used for direct visualization via the creation of bone and tumor models. ${ }^{13,28,29)}$ However, virtual modeling is more common than real 3D-printed models and is utilized to design PSI and custommade implants (Graphical abstract). ${ }^{18-20,22,30)}$

\section{Patient-specific instruments}

In orthopedics, traditional cutting blocks or surgical guides for arthroplasty are non-patient-specific multiuse instruments. Many commercial PSI are utilized to enhance bone-cutting accuracy and reaming for arthroplasty. The PSI for conventional arthroplasty implants has a patient-specific shape but match each company's commercial implant. However, in orthopedic oncology, PSI are utilized to ensure the accuracy of wide excision or fitting 3D-printed custom-made implants to bone defects. Implant fitting for massive bone defects is possible under the assumption of planned bone cutting. Therefore, the PSI in orthopedic oncology is designed with a 3D-printed implant.

Bone-cutting accuracy is the most important outcome of guide use. This often-reported problem is a major pitfall in PSI for arthroplasty. ${ }^{31)}$ Compared to other orthopedic surgeries, limb salvage surgery has advantages in this respect. Limb salvage surgery for sarcomas generally requires greater surgical exposure than other types of orthopedic surgeries. A wide opening in the surgical field can provide clear visualization of the bony landmarks and a sufficient contact surface of the PSI to the bone, thus reducing the possibility of PSI malpositioning. Bonecutting accuracy by a PSI in orthopedic oncology is compatible to navigation system use, ${ }^{32)}$ and Park et al. ${ }^{14)}$ reported maximal bone-cutting errors using a 3D-printed PSI of $3 \mathrm{~mm}$ and mean shortest and longest bone-cutting errors of $1.2 \mathrm{~mm}$ and 1.4 $\mathrm{mm}$, respectively, of normal bones surrounding the tumor. Considering that the planned normal bone length surrounding the bone tumor is usually set to $1-2 \mathrm{~cm}$ or more in the wide excision of bone tissue, an error of even several millimeters may not adversely affect the oncologic margin status. Depending on the situation, the contact surface can be easily expanded with the closed-type PSI, or it is sometimes advantageous to deliberately use the open-type PSI. Notably, it is more important to fabricate

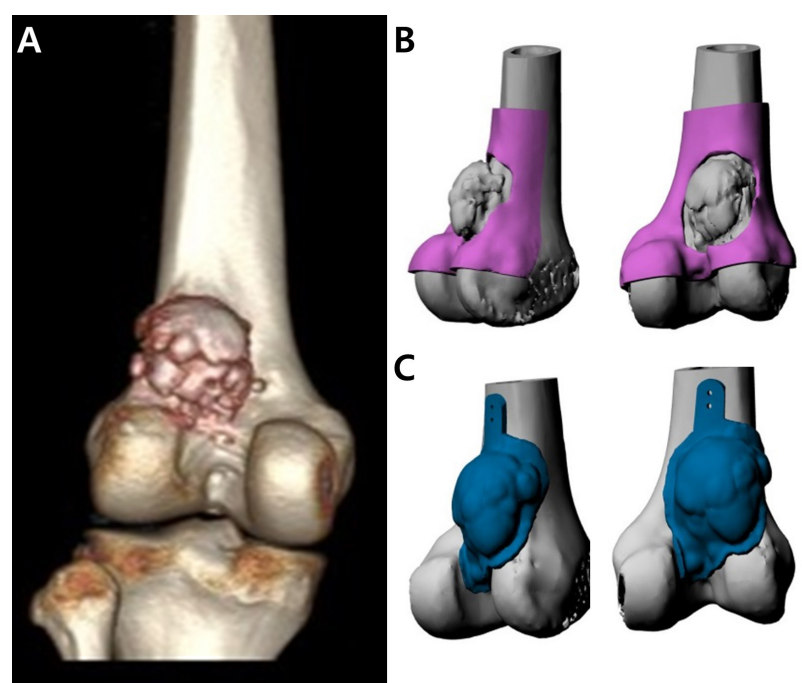

Fig. 2. Open and closed type of 3-dimensional printing bone-tumor cutting guide as personalized surgical instrument. For a patient with periosteal osteosarcoma of the distal femur (A), open (B) and closed (C) type bone-tumor cutting guides were designed. ${ }^{16)}$

a PSI while avoiding contact with soft-tissue expansion of the tumor or tough soft tissues that are difficult to remove (Fig. 2). ${ }^{14,17)}$

\section{Custom-made implants}

Limb salvage surgery includes wide excision for bone sarcoma to enable bone and soft-tissue reconstruction. Wide excision for bone sarcoma often results in large bone defects, which have unique features in each patient. In other words, anatomical location, bone defect size and shape, adjacent joint involvement, and soft-tissue loss around the bone defect differ and require a personalized reconstruction strategy. One of the important aspects of limb salvage surgery is bone defect filling. Commercially available megaprosthesis is the most commonly used surgical option, but implant size and the applicable anatomical location are limited, and it is often difficult to use in children due to size mismatch. Structural bone allografts and recycled autografts are feasible options, but weak mechanical strength and osteolysis are major problems over time.

Metal 3D printing recently became feasible, and 3D-printed implants have been utilized to reconstruct massive bone defects after wide excision. ${ }^{12,15-22)}$ The major advantages of using 3Dprinted implants are their "custom-made" nature. The concept of custom-made implants is not new, as these prostheses have been used reported for pelvic reconstruction until just recently. However, long fabrication times and high expense are major limitations. ${ }^{33,34)}$ As 3D-printing technology overcomes these limitations, custom-made implants are re-emerging. Regardless of bone and tumor size and location, implants can be fabricated in a few weeks by $3 \mathrm{D}$ printing. Moreover, unlike the previous custom-made implant that simply focuses on filling bone defects, 3D-printed custom-made implants attempt functional reconstruction by hybridization with conventional orthopedic implants or providing scaffolds for tissue integration. ${ }^{19,21)}$ When 
bone tumor occurs close to the joint, the joint is often sacrificed for conventional tumor replacement implants for implant fixation because of the short normal bone stock. However, using custommade implants, it is possible to save the joint that otherwise is lost due to being close to the bone tumor. Moreover, surgical time may be reduced compared to those other reconstruction options because 3D implants are already matched with the bone defects; therefore, processes such as allograft shaping can be omitted, and implant fixation is simply performed as planned without requiring processes such as plate bending. A direct comparison of the surgical time was not reported because of the variety of bone-tumor surgeries. For example, for bone-tumor surgery of the same pelvic bone, the surgery time may range from less than 2 hours to more than 10 hours depending on tumor size, surgical extent, bone reconstruction method, and artificial joint inclusion status. Park et al. ${ }^{20)}$ reported that reconstruction during limb salvage surgery using a 3D implant took 15-220 minutes, while the pelvic and long bones took 81-108 minutes, respectively. Liang et al. ${ }^{18)}$ and Wang et al. ${ }^{35)}$ reported 258 and 271 minutes for the whole procedure, including wide excision and reconstruction, respectively. The reconstruction time appears to have been shortened using 3D implants. Using 3D printed implants, surgeons can focus more on complete tumor resection by reducing the burden of reconstruction. Instead, it is necessary to spend significant preoperative time printing an optimized design and implant.

Clinical outcomes and complications after limb salvage surgery using custom-made 3D-printed implants have been reported in a few studies owing to the novelty of the 3D-printing technique. To date, several case series have reported promising surgical outcomes. Anatomical location seems to be the most important factor in terms of complications. The complication rate was reportedly high for pelvic reconstruction; in fact, it was the same as that of conventional implants. Compared to limb salvage surgery using conventional implants, 3D-implant surgery does not cause unexpected new complications or a high complications rate. However, long-term results are needed to properly evaluate clinical outcomes and complications (Table 1). ${ }^{15,18-20,35-39)}$

Concerns about 3D implants are longevity related to the mechanical properties of the metal products made by a new fabrication method, metal powder-based AM. Clinical evidence for implant longevity is limited in previous studies due to the short follow-up period. ${ }^{15-20,22)}$ A solid 3D-printed structure made of Ti6Al4V has equivalent ultimate tensile strength, yield strength, and elongation. ${ }^{740)}$ Its fatigue properties, which are related to its surface roughness, unwanted porosity-related defects, building orientation, postprocessing such as machining, and hot isostatic pressing, require further study. ${ }^{41)}$ The mesh structure refers to a form with intentional internal porosity with a repeating pattern that can be fabricated only by AM. A mesh structure is utilized as a part of the implant body in orthopedic oncology and as a surface coating of cementless knee and hip arthroplasty implants. ${ }^{42,43)}$ This provides a scaffold for tissue integration and reduces the stress-shielding effect. However, the mesh structure is mechanically weak, and an implant fracture may be triggered at the interface between the solid and mesh structures. $^{211}$

\section{Tissue engineering}

Tissue engineering in orthopedic surgery is currently experimental. Skin, vessels, cartilage, and bone tissue are common targets in orthopedics. ${ }^{544-49)}$ In addition, bioprinting has advantages over traditional methods for $3 \mathrm{D}$ cell culture for creating and utilizing a tumor model. ${ }^{8)}$

\section{Case presentation}

\section{Preoperation}

A 14-year-old girl presented with left knee pain. Plain radiographs showed a bone tumor with a mixed pattern but no clear tumor margin. Magnetic resonance imaging (MRI) showed that the tumor was located over the medial epiphysis and metaphysis of the proximal tibia (Fig. 3). Positron emission tomography/ computed tomography (CT) showed no distant metastasis. Two cycles of neoadjuvant chemotherapy were administered before limb salvage surgery was performed. During chemotherapy, a 3D-printed implant was designed for the proximal tibia.

\section{Design and manufacturing of 3D-printed implant}

CT and MRI images of the proximal tibia were used to construct a 3D bone and tumor model using commercial bone

Table 1. Clinical reports of 3-dimensional (3D)-printed custom-made implants in orthopedic oncology

\begin{tabular}{|c|c|c|c|c|}
\hline Study & No. of patients & Follow-up (mo) & Anatomical site & Complications ( $\mathrm{n}$ ) \\
\hline Liang et al., 2017 & 35 & 20.5 (mean) & Pelvis & $9 / 35^{a)}$ \\
\hline Wang et al., 2018 35$)$ & 11 & 15.5 (mean) & Pelvis & $3 / 11^{\mathrm{b})}$ \\
\hline Angelini et al., 20195) $2020^{36)}$ & 41 & 20 (mean) & Various, Mainly pelvis & $9 / 41^{c)}$ \\
\hline Lu et al., 201937) & 10 & $5-34$ & Femur, tibia $\left.\left(5 \mathrm{PFB}^{\mathrm{d}}\right), 5 \mathrm{~PB}^{\mathrm{e}}\right)$ & $0 / 10$ \\
\hline Park et al., $2020^{19)}$ & 10 & $7-33$ & Various & $0 / 10$ \\
\hline Park et al., $2021^{20)}$ & 12 & $3-28$ & Pelvis & $0 / 12$ \\
\hline Jovičić et al., 2021 38 ) & 11 (10 children) & 33 (mean) & Various Mainly humerus & $6 / 11^{f)}$ \\
\hline Beltrami et al., $2021^{39)}$ & 11 Children & 25.7 & Various & $4 / 11^{9)}$ \\
\hline
\end{tabular}

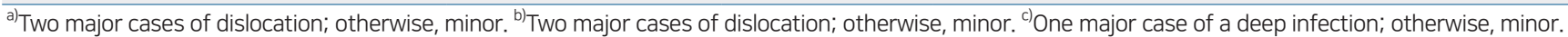
d) PFB, 3D-printed prosthesis + vascularized fibula + bioceramic granules. ${ }^{\mathrm{e}} \mathrm{PB}, 3 \mathrm{D}$-printed prosthesis + bioceramic granules. ${ }^{f)}$ Four major cases of dislocation $(n=3)$ and compartment syndrome $(n=1)$; otherwise, minor. ${ }^{g}$ One major case of deep infection; otherwise, minor. 

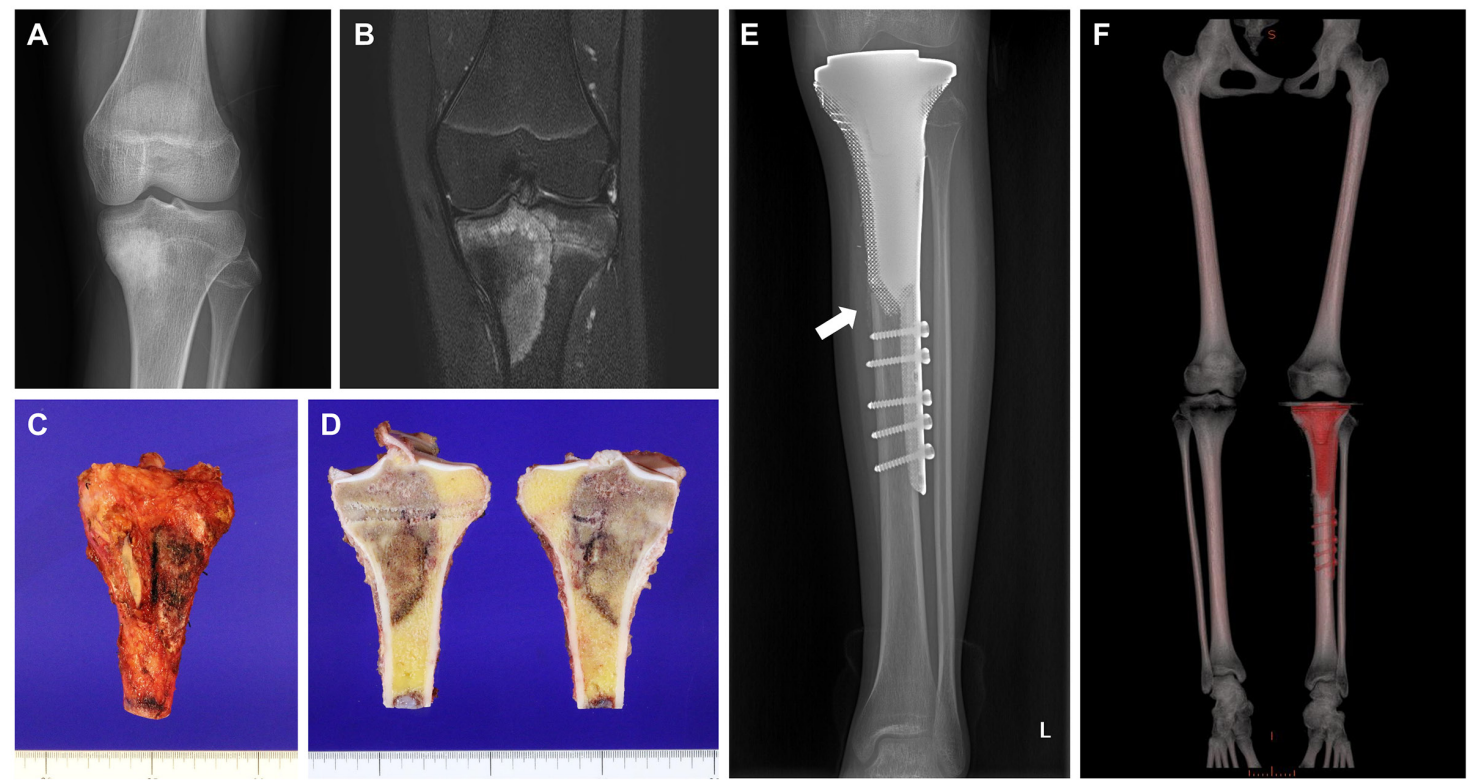

Fig. 3. Case presentation of a 14-year-old girl with osteosarcoma of the proximal tibia. A plain radiograph (A) and T2-weighted magnetic resonance image (B) showed typical osteosarcoma of the proximal tibia. Photographs of the gross specimen (C) and a cross-section of the specimen (D) showing that a wide excision was completed as planned with sufficient margins. A postoperative plain radiograph $(E)$ and computed tomography reconstruction image $(F)$ presented bone reconstruction with the 3-dimensional-printed implant. The arrow indicates callus formation.

A

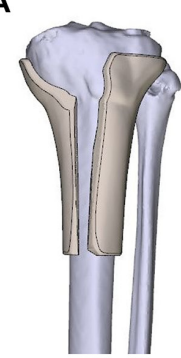

C

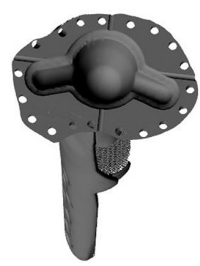

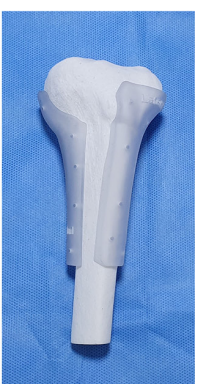

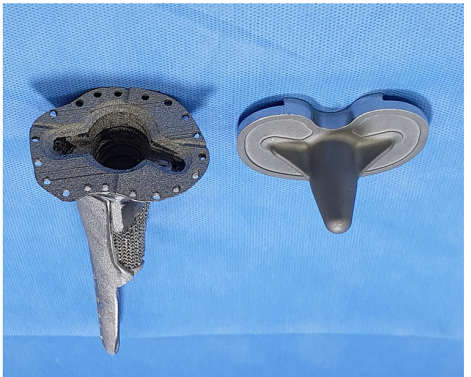

B

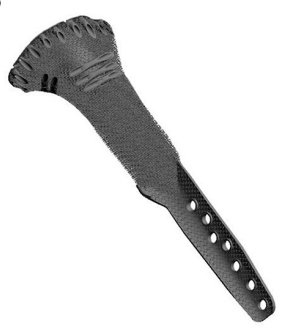

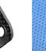

D
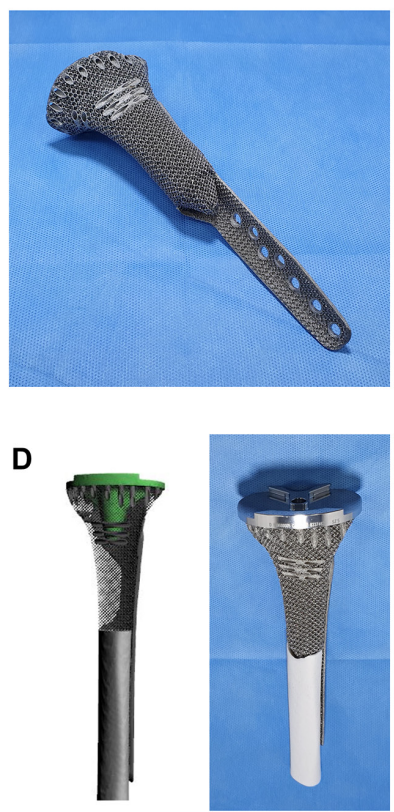

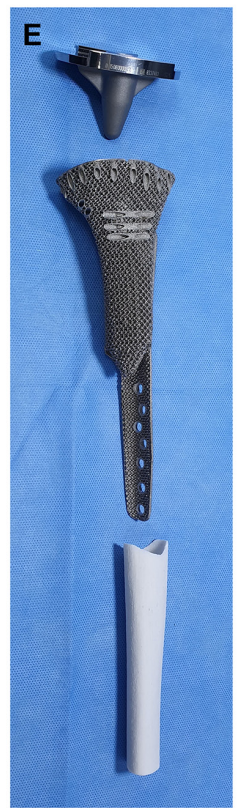

Fig. 4. Designs of the 3-dimensionally (3D)-printed products. Three-dimensional designs and photographs for the 3D-printed personalized surgical instrument (A) and the implant (B) are shown. (C) A design and photograph for the proximal tibial surface shows a matching socket for the tibial implant of conventional knee arthroplasty. (D, E) A design and photograph showing the $3 \mathrm{D}$ implant conjugated with the conventional knee arthroplasty implant.

modeling software (MIMCS, Materialize, Belgium). For the specific implant design, an orthopedic surgeon and an engineer are required to contribute and communicate. In terms of clinical considerations, we attempted to maintain the distal femur with growth potential and planned hemiarthroplasty for the proximal tibia only. We planned to insert only the femoral component in a future revision total knee arthroplasty. Intra-articular tumor invasion was not observed, and the distal bone margin for wide excision was set at $3 \mathrm{~cm}$. PSI were designed to achieve the planned margins. To articulate, a conventional total knee arthroplasty implant was utilized in conjugation with a 3D-printed custommade implant for the proximal tibia. The 3D-printed implant had a shape complementary to that of the conventional knee arthroplasty implant, with multiple holes for proximally suturing the patellar tendon and knee joint capsule. At the distal end of the implant, the implant body was transformed into a lateral plate for screw fixation of the distal tibia. The final implant was 196 mm long and weighed $153 \mathrm{~g}$ (Fig. 4). 


\section{Surgery with 3D-printed proximal tibial implant}

With the patient in the supine position, a curved incision was made from the distal thigh to the lower leg. The patellar tendon was detached from the tibial insertion, and the soft tissues around the proximal tibia were dissected with a safe margin. A knee joint capsulotomy was performed, the cruciate ligaments were cut, and the tibia was completely separated from the distal femur along with the medial and lateral menisci. A tibial shaft osteotomy was performed using bone-cutting guides. The matched socket design of the conventional arthroplasty implant enabled simultaneous conjugation of the 3D-printed implant and tibial plate outside the surgical field during surgery. A 3D-printed custom-made cone-shaped reamer was used to enhance the bone-implant contact surface. The assembled implant (the 3D-printed implant and the tibial component of the conventional total knee arthroplasty implant) was placed in the bone defect, and the fitting was grossly perfect. The knee joint capsule and patellar tendon were sutured using premade suture holes in the 3Dimplant. Radiation exposure was minimized because the 3D-printed cutting guide eliminated the need for C-arm fluoroscopy during surgery.

\section{Postoperative course}

No adverse events occurred during the postoperative period. The suction drain for the surgical site was removed at 1 week postoperative, and intravenous antibiotics were continued over time. Adjuvant chemotherapy for osteosarcoma was resumed at 3 weeks postoperative. From 1 week postoperative, continuous passive knee motion was allowed and gradually increased to 90 degrees, and crutch walking with a knee brace was started. At the last follow-up 9 months after the index surgery, the patient was able to walk unsupported without limping (Fig. 4).

\section{Conclusion}

Three-dimensional printing technology is more actively utilized in orthopedic oncology than in other orthopedic fields. The 3D-printed implant can replace the conventional tumor prosthesis and auto/allobone graft, enabling personalized bone reconstruction. Currently, only a single titanium alloy material is practically used, but biodegradable materials or bioprinted materials will become available. Thus, biologic reconstruction using $3 \mathrm{D}$ techniques will be possible in the future.

\section{Ethical consideration}

The study was conducted after approval was obtained from the Institutional Review Board of National Cancer Center (NCC2017-0129). The presenting patient provided written informed consent prior to undergoing surgery after a thorough explanation of the surgical options.
See the commentary on "Application of 3-dimensional printing implants for bone tumors" via https://doi.org/10.3345/ cep.2022.00080.

\section{Footnotes}

Conflicts of interest: No potential conflict of interest relevant to this article was reported.

Funding: This study was supported by the National Cancer Center Grant (No. NCC-2110270).

ORCID:

Jong Woong Park @ https://orcid.org/0000-0002-8044-7624

Huyn Guy Gang @ https://orcid.org/0000-0003-1994-6990

Author contribution: Conceptualization: PJW, KHG; Data Curation: Not applicable; Formal analysis: Not applicable; Funding acquisition: PJW, KHG; Methodology: PJW, KHG; Project administration: PJW, KHG; Visualization: PJW, KHG; Writing-original draft: PJW; Writing-review\&editing: PJW, KHG

\section{References}

1. Kodama H. Automatic method for fabricating a three-dimensional plastic model with photo-hardening polymer. Rev Sci Instrum 1981;52:1770-3.

2. Nagarajan N, Dupret-Bories A, Karabulut E, Zorlutuna P, Vrana NE. Enabling personalized implant and controllable biosystem development through 3D printing. Biotechnol Adv 2018;36:521-33.

3. Pucci JU, Christophe BR, Sisti JA, Connolly ES Jr. Three-dimensional printing: technologies, applications, and limitations in neurosurgery. Biotechnol Adv 2017;35:521-9.

4. Kaye R, Goldstein T, Zeltsman D, Grande DA, Smith LP. Three dimensional printing: a review on the utility within medicine and otolaryngology. Int J Pediatr Otorhinolaryngol 2016;89:145-8.

5. Murphy SV, Atala A. 3D bioprinting of tissues and organs. Nat Biotechnol 2014;32:773-85.

6. Jamroz W, Szafraniec J, Kurek M, Jachowicz R. 3D Printing in pharmaceutical and medical applications - recent achievements and challenges. Pharm Res 2018;35:176.

7. Liu S, Shin YC. Additive manufacturing of Ti6Al4V alloy: a review. Mat Design 2019; 164:107552.

8. Li M, Song X, Jin S, Ye K. 3D tumor model biofabrication. Bio-Des Manuf 2021;4:526-40.

9. Berman B. 3-D printing: the new industrial revolution. Bus Horiz 2012; 55:155-62.

10. Weller C, Kleer R, Piller FT. Economic implications of 3D printing: market structure models in light of additive manufacturing revisited. Int J Prod Econ 2015;164:43-56.

11. Kim Y, Jang WY, Park JW, Park YK, Cho HS, Han I, et al. Transepiphyseal resection for osteosarcoma in patients with open physes using MRI assessment. Bone Joint J 2020;102-B:772-8.

12. Kang HG. Clinical atlas of $3 \mathrm{D}$ printing bone reconstruction. Singapore: Springer, 2021.

13. McCulloch RA, Frisoni T, Kurunskal V, Maria Donati D, Jeys L. Computer navigation and $3 \mathrm{D}$ printing in the surgical management of bone sarcoma. Cells 2021;10:195 
14. Park JW, Kang HG, Lim KM, Park DW, Kim JH, Kim HS. Bone tumor resection guide using three-dimensional printing for limb salvage surgery. J Surg Oncol 2018;118:898-905.

15. Angelini A, Trovarelli G, Berizzi A, Pala E, Breda A, Ruggieri P. Threedimension-printed custom-made prosthetic reconstructions: from revision surgery to oncologic reconstructions. Int Orthopaed 2019;43:12332.

16. Ji T, Yang Y, Tang X, Liang H, Yan T, Yang R, et al. 3D-printed modular hemipelvic endoprosthetic reconstruction following periacetabular tumor resection: early results of 80 consecutive cases. J Bone Joint Surg Am Vol 2020;102:1530-41.

17. Kang HG, Park JW, Park DW. Three dimensional printing technique and its application to bone tumor surgery. J Korean Orthopaed Assoc 2018;53:466.

18. Liang H, Ji T, Zhang Y, Wang Y, Guo W. Reconstruction with 3D-printed pelvic endoprostheses after resection of a pelvic tumour. Bone Joint J 2017;99-B:267-75.

19. Park JW, Kang HG, Kim JH, Kim HS. New 3-dimensional implant application as an alternative to allograft in limb salvage surgery: a technical note on 10 cases. Acta Orthopaed 2020;91:489-96.

20. Park JW, Kang HG, Kim JH, Kim HS. The application of 3D-printing technology in pelvic bone tumor surgery. J Orthop Sci 2021;26:276-83.

21. Park JW, Shin YC, Kang HG, Park S, Seo E, Sung H, et al. In vivo analysis of post-joint-preserving surgery fracture of 3D-printed Ti-6Al-4V implant to treat bone cancer. Bio-Des Manuf 2021;4:879-88.

22. Wong KC, Kumta SM, Geel NV, Demol J. One-step reconstruction with a 3D-printed, biomechanically evaluated custom implant after complex pelvic tumor resection. Comput Aided Surg 2015;20:14-23.

23. Won SH, Lee YK, Ha YC, Suh YS, Koo KH. Improving pre-operative planning for complex total hip replacement with a rapid prototype model enabling surgical simulation. Bone Joint J 2013;95-B:1458-63.

24. Shen S, Wang P, Li X, Han X, Tan H. Pre-operative simulation using a three-dimensional printing model for surgical treatment of old and complex tibial plateau fractures. Sci Rep 2020;10:6044.

25. Paramasivam V, Singh G, Santhanakrishnan S. 3D printing of human anatomical models for preoperative surgical planning. Procedia Manuf 2020;48:684-90.

26. Choi SW, Park KS, Yoon TR. Patient-specific three-dimensional bone model for the diagnosis and treatment of orthopedic diseases of the hip: opinions of orthopedic surgeons on new medical technology. Hip Pelvis 2021;33:96-101.

27. Calvo-Haro JA, Pascau J, Mediavilla-Santos L, Sanz-Ruiz P, SanchezPerez C, Vaquero-Martin J, et al. Conceptual evolution of 3D printing in orthopedic surgery and traumatology: from "do it yourself" to "point of care manufacturing." BMC Musculoskelet Disord 2021;22:360.

28. George E, Barile M, Tang A, Wiesel O, Coppolino A, Giannopoulos A, et al. Utility and reproducibility of 3-dimensional printed models in pre-operative planning of complex thoracic tumors. J Surg Oncol 2017;116:407-15.

29. Kim MP, Ta AH, Ellsworth WA 4th, Marco RA, Gaur P, Miller JS. Three dimensional model for surgical planning in resection of thoracic tumors. Int J Surg Case Rep 2015; 16:127-9.

30. Gouin F, Paul L, Odri GA, Cartiaux O. Computer-assisted planning and patient-specific instruments for bone tumor resection within the pelvis: a series of 11 patients. Sarcoma 2014;2014:842709.

31. Hafez MA, Moholkar K. Patient-specific instruments: advantages and pitfalls. SICOT J 2017;3:66.

32. Wong KC, Sze KY, Wong IO, Wong CM, Kumta SM. Patient-specific instrument can achieve same accuracy with less resection time than navigation assistance in periacetabular pelvic tumor surgery: a cadaveric study. Int J Computer Assist Radiol Surgery 2016;11:307-16.

33. Kotz R. The history of bone tumour treatment and the state of the art in Vienna. Pril (Makedon Akad Nauk Umet Odd Med Nauki) 2017;38:31-
8.

34. Sim FH, Choong PF, Weber KL. Master techniques in orthopaedic surgery: orthopaedic oncology and complex reconstruction. Philadelphia (PA): Lippincott Williams \& Wilkins, 2011.

35. Wang B, Hao Y, Pu F, Jiang W, Shao Z. Computer-aided designed, three dimensional-printed hemipelvic prosthesis for peri-acetabular malignant bone tumour. Int Orthop 2018;42:687-94.

36. Angelini A, Kotrych D, Trovarelli G, Szafrański A, Bohatyrewicz A, Ruggieri P. Analysis of principles inspiring design of three-dimensionalprinted custom-made prostheses in two referral centres. Int Orthopaed 2020;44:829-37.

37. Lu Y, Chen G, Long Z, Li M, Ji C, Wang F, et al. Novel 3D-printed prosthetic composite for reconstruction of massive bone defects in lower extremities after malignant tumor resection. J Bone Oncol 2019;16: 100220.

38. Jovičić M, Vuletić F, Ribičić T, Šimunić S, Petrović T, Kolundžić R. Implementation of the three-dimensional printing technology in treatment of bone tumours: a case series. Int Orthopaed 2021;45:107985 .

39. Beltrami G, Ristori G, Nucci AM, Galeotti A, Tamburini A, Scoccianti $\mathrm{G}$, et al. Custom-made 3D-printed implants as novel approach to reconstructive surgery after oncologic resection in pediatric patients. J Clin Med 2021;10:1056.

40. Galarraga H, Lados DA, Dehoff RR, Kirka MM, Nandwana P. Effects of the microstructure and porosity on properties of Ti-6Al-4V ELI alloy fabricated by electron beam melting (EBM). Addit Manuf 2016;10:4757.

41. Chern AH, Nandwana P, Yuan T, Kirka MM, Dehoff RR, Liaw PK, et al. A review on the fatigue behavior of Ti-6Al-4V fabricated by electron beam melting additive manufacturing. Int J Fatigue 2019;119:173-84.

42. Geng X, Li Y, Li F, Wang X, Zhang K, Liu Z, et al. A new 3D printing porous trabecular titanium metal acetabular cup for primary total hip arthroplasty: a minimum 2-year follow-up of 92 consecutive patients. J Orthop Surg Res 2020;15:383.

43. Sultan AA, Mahmood B, Samuel LT, Stearns KL, Molloy RM, Moskal JT, et al. Cementless 3D printed highly porous titanium-coated baseplate total knee arthroplasty: survivorship and outcomes at 2-year minimum follow-up. J Knee Surg 2020;33:279-83.

44. Arguchinskaya NV, Beketov EE, Kisel AA, Isaeva EV, Osidak EO, Domogatsky SP, et al. The technique of thyroid cartilage scaffold support formation for extrusion-based bioprinting. Int J Bioprint 2021;7:348.

45. Choudhury D, Anand S, Naing MW. The arrival of commercial bioprinters - towards 3D bioprinting revolution! Int J Bioprint 2018;4: 139.

46. Gu Z, Fu J, Lin H, He Y. Development of 3D bioprinting: from printing methods to biomedical applications. Asian J Pharm Sci 2020;15:529-57.

47. Jang MJ, Bae SK, Jung YS, Kim JC, Kim JS, Park SK, et al. Enhanced wound healing using a 3D printed VEGF-mimicking peptide incorporated hydrogel patch in a pig model. Biomed Mater 2021;16:045013.

48. Ma H, Li T, Huan Z, Zhang M, Yang Z, Wang J, et al. 3D printing of highstrength bioscaffolds for the synergistic treatment of bone cancer. NPG Asia Materials 2018;10:31-44.

49. Zhu W, Ma X, Gou M, Mei D, Zhang K, Chen S. 3D printing of functional biomaterials for tissue engineering. Curr Opin Biotechnol 2016;40:103-12.

How to cite this article: Park JW, Kang HG. Application of 3-dimensional printing implants for bone tumors. Clin Exp Pediatr 2022;65:476-81. https://doi.org/10.3345/cep.2021. 01326 